SCIENTIFIC CORRESPONDENCE

\title{
Inflamed juvenile conjunctival naevus: clinicopathological characterisation
}

\author{
Ehud Zamir, Hadas Mechoulam, Alessandra Micera, Francesca Levi-Schaffer, \\ Jacob Pe'er
}

Br J Ophthalmol 2002;86:28-30

Aim: Inflamed juvenile conjunctival naevi (IJCN) are often erroneously suspected to be malignant because of rapid growth. Their clinical and histopathological features have not been characterised in series of patients. The aim of the study is to characterise IJCN clinically and histopathologically.

Methods: This is a retrospective non-randomised clinicopathological study. All patients younger than 20 years with conjunctival naevi which were excised between 1990 and 2000 were included. The clinical signs of the affected patients and the histopathological findings of the excised lesions were characterised.

Results: A total of 63 conjunctival naevi were resected. $25 \%$ of the patients had simple compound conjunctival naevi and $75 \%$ had compound naevi with prominent inflammatory histological features (discrete lymphocyte aggregates, plasma cells, and eosinophils). Epithelial cysts and solid epithelial islands were common in the IJCN. The IJCN were all located at or near the limbus, and characterised by recurrent periods of congestion and growth. 75\% of the IJCN patients with complete medical records had a history of allergic disease. Marked conjunctival papillary reaction was present in all of the patients, indicating a possible conjunctival allergy.

Conclusions: IJCN is a unique entity, different from simple compound conjunctival naevus. Its association with allergic conjunctivitis is suggestive, and despite periods of alarmingly rapid growth, is histologically benign.

C onjunctival naevi are common lesions of the conjunctival epithelium and substantia propria. They are classified as either congenital or acquired, and usually develop during the first decade of life. Acquired naevi are typically located near the limbus, and may drape over the peripheral cornea. ${ }^{1}$ They rarely require excision, except where rapid growth or irritative symptoms are present.

"Inflamed naevi of puberty and young adulthood," as termed by Folberg and colleagues in their review of benign conjunctival melanocytic lesions, ${ }^{1}$ are conjunctival naevi that seem to be enlarging and are histologically associated with extensive inflammatory infiltrate. It is our impression that these rapidly enlarging naevi are often approached with undue concern by patients and clinicians, usually suspecting malignancy. Our aim was to study the clinical and histological characteristics of this entity.

\section{METHODS}

We retrospectively reviewed the clinical charts of patients under the age of 20 years who underwent surgical removal of conjunctival lesions between 1990 and 2000. Patients whose specimens were processed in our ocular pathology laboratory, and who had a histopathological diagnosis of conjunctival naevus, were selected. Pertinent ocular and, when available, systemic history was summarised. We then reviewed the histopathological sections (formalin fixed, paraffin embedded, $5 \mathrm{~mm}$ sections, haematoxylin and eosin and periodic acid Schiff stains). The diagnosis of inflamed conjunctival naevus was made in cases of compound naevi that had prominent inflammatory cellular infiltration within their subepithelial tissue.

\section{RESULTS}

We found a total of 63 specimens of conjunctival naevi that were excised during the study period from patients under age 20 . Sixteen specimens (25\%) (median age of patients 14 years, mean age 13.5 (SD 5.06) years) were histologically defined as simple, compound conjunctival naevi, while 47 (75\%) (median age of patients 13 years, mean age 12.7 (4.2) years) were inflamed lesions (IJCN). Fifty three per cent of patients were males, while $47 \%$ were females. The clinical and histological characteristics of the IJCN lesions are summarised in Table 1.

All lesions were juxtalimbal (Fig l (1-3)); 21 (45\%) were pigmented (Fig l (2)). All patients were referred because of a history of moderate or rapid "growth" or congestion of the conjunctival lesion for several weeks to several months, in some cases with increased pigmentation. Some of the patients reported use of anti-inflammatory drops, with temporary decrease in the congestion of the lesion. Eighteen of the 24 patients with complete systemic records had a history of various allergic diseases, including vernal conjunctivitis. In all IJCN patients, examination of the eyes showed obvious papillary reaction that indicated allergic conjunctivitis. In none of

Table 1 Clinical and histopathological features of the inflamed juvenile conjunctival naevi

\begin{tabular}{|c|c|c|}
\hline & No & $\%$ \\
\hline \multicolumn{3}{|l|}{ Clinical features } \\
\hline Juxtalimbal lesion location & $47 / 47$ & 100 \\
\hline Clinically pigmented lesions & $21 / 47$ & 45 \\
\hline $\begin{array}{l}\text { History of allergy/atopy or "vernal } \\
\text { conjunctivitis" (complete systemic } \\
\text { history available only in } 24 \text { records) }\end{array}$ & $18 / 24$ & 75 \\
\hline \multicolumn{3}{|l|}{ Histopathological features } \\
\hline Epithelial cysts in the substantia propria & $32 / 47$ & 68 \\
\hline $\begin{array}{l}\text { Solid epithelial islands in the substantia } \\
\text { propria }\end{array}$ & $34 / 47$ & 72 \\
\hline Either solid or cystic epithelial elements & $42 / 47$ & 89 \\
\hline Microscopic pigmentation & $12 / 47$ & 25 \\
\hline Junctional nests & $47 / 47$ & 100 \\
\hline Lymphocyte infiltration & $47 / 47$ & 100 \\
\hline Germinal centres & $14 / 47$ & 30 \\
\hline Plasma cell infiltration & $32 / 47$ & 68 \\
\hline Eosinophil infiltration & $36 / 47$ & 77 \\
\hline
\end{tabular}



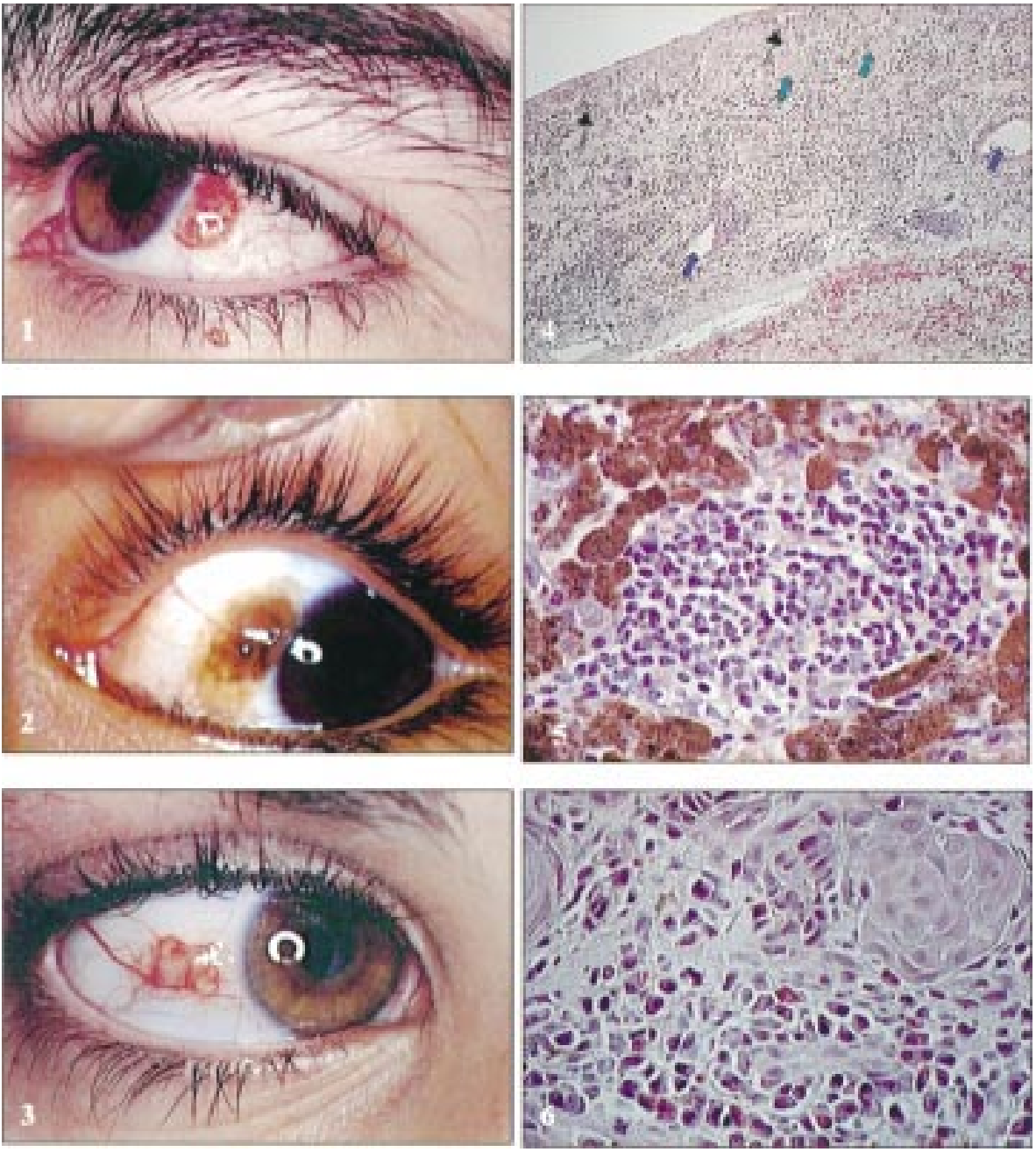

Figure 1 (1) A 12 year old boy with bilateral vernal conjunctivitis. This markedly congested and mildly elevated conjunctival cystic lesion was removed after 3 years of gradual growth. (2) A 14 year old boy with a juxtalimbal, pigmented conjunctival lesion adjacent to the superotemporal limbus. The lesion was known since infancy, but accelerated growth started at age 13. Intralesional cysts are visible clinically. (3) A 13 year old girl with allergic conjunctivitis and a rapidly growing, amelanotic, juxtalimbal lesion, with congested "feeder vessels." (4) Low magnification histopathological section. Notice naevus cells with junctional activity (examples indicated by black arrows), solid epithelial islands (green arrows), and cystic epithelial islands (blue arrows). Diffuse, chronic inflammatory infiltration is seen in the conjunctival stroma (haematoxylin and eosin, $\times 10$ ). (5) High magnification histopathological section of the lesion seen in (2). An island of lymphocytes is surrounded by pigmented cells (haematoxylin and eosin, $\times 100$ ). (6) High magnification histopathological section showing numerous eosinophils and plasma cells. Notice an epithelial island on the right upper part (haematoxylin and eosin, $\times 100$ ).

the patients with simple conjunctival naevus was a history of allergy or conjunctival papillary reaction recorded.

In histopathological examination, in 42 of the 47 lesions $(89 \%)$ we found cystic or solid epithelial elements, in 68\% epithelial cysts, and in $72 \%$ solid epithelial islands (Fig l $(4,6)$ ). In all lesions there were significant lymphocytic infiltrations (Fig $1(4,5))$, in $14(30 \%)$ germinal centres were found, in 32 $(68 \%)$ plasma cells were found (Fig l (6)), and in $36(77 \%)$ significant infiltration of eosinophils was noticed (Fig 1 (6)).

\section{DISCUSSION}

Inflamed juvenile conjunctival naevi have not been characterised in clinicopathological series so far. This series demonstrates that although clinically alarming, these rapidly growing lesions do not differ from simple, compound conjunctival naevi in their benign histopathological features. These lesions are more likely to be excised than simple naevi, as in our series, because of their relatively rapid growth and congestion. As a rule, malignant conjunctival melanomas are exceedingly rare during childhood, while naevi are common. $^{1-4}$ Periods of rapid growth of inflamed naevi represent inflammatory infiltration and cystic enlargement, rather than malignant proliferation.

Some clinical and histological features are unique to IJCN. Unlike simple compound naevus, they usually show periods of inflammatory reaction and are surrounded by vascular congestion.

The high incidence of allergy, allergic conjunctivitis, or vernal conjunctivitis is interesting. These children would typically be treated for allergic or vernal conjunctivitis for varying periods of time before rapid growth of the naevus was noted. It may be that chronic allergic conjunctival inflammation, through the release of various mediators and notably of stem cell factor by conjunctival mast cells ${ }^{5}$ and infiltrating eosinophils, ${ }^{6}$ incites the development of the naevus, with subsequent periods of rapid growth. Histologically, eosinophils were abundant in three quarters of the specimens, and several partially degranulated mast cells were also noticed in these conjunctival biopsies (unpublished observation). Conjunctival epithelial components (either cysts or solid epithelial elements) are classically reported in $50 \%$ of conjunctival naevi. ${ }^{7}$ We found microscopic cysts in $68 \%$ of specimens, solid epithelial islands in $72 \%$ of the specimens, and $89 \%$ of patients had 
either cystic or solid epithelial elements in the naevus. Clinically evident intralesional cysts should therefore help in the diagnosis of inflamed naevi.

We believe that typical cases of IJCN can be differentiated on clinical grounds from malignant conjunctival melanomas. It is worth noting that IJCN should also be differentiated from "salmon patch" lesions of conjunctival lymphoma, which is exceedingly rare in childhood. A pink, elevated lesion of the conjunctiva at a young age, especially at the limbus, is more likely to be an IJCN lesion than either a melanoma or lymphoma.

The patient's young age and the cystic nature of typical lesions are indicators of a benign lesion. Although in patients with such typical lesions observation alone may suffice, we would recommend excisional biopsy of atypical lesions or whenever the clinician cannot make a definite diagnosis of IJCN. Similarly, lesions causing functional problems, such as dellen, interference with contact lens wear, or a significant cosmetic blemish should probably be excised. If the patient or his parents seem concerned about the lesion, it is also probably best to recommend excision.

In summary, IJCN is a benign, usually juxtalimbal, and mostly amelanotic conjunctival naevus that may show rapid growth. It is usually found in patients with chronic allergic conjunctivitis, and the clinical and histological findings associated with this naevus are of obvious allergic nature. Ophthalmologists should be aware of the benign nature of this entity, and not confuse it with conjunctival malignant melanoma, which is so rare in this age group.

\section{ACKNOWLEDGMENTS}

This work was presented in part at the ARVO meeting in May 2000. This study was supported in part by a grant from the Aimwell Trust (UK).

\section{Authors' affiliations}

E Zamir, H Mechoulam, J Pe'er, Department of Ophthalmology, Hadassah University Hospital, Jerusalem, Israel

A Micera, F Levi-Schaffer, Department of Pharmacology, School of Pharmacy, Faculty of Medicine, The Hebrew University, Jerusalem, Israel

Correspondence to: Jacob Pe'er, MD, Department of Ophthalmology, Hadassah University Hospital, PO Box 12000, 91120 Jerusalem, Israel; peer@md2.huji.ac.i

Accepted for publication 7 June 2001

\section{REFERENCES}

1 Folberg $\mathbf{R}$, Jakobiec FA, Bernardino VB, et al. Benign conjunctival melanocytic lesions. Clinicopathologic features. Ophthalmology 1989;96:436-61

2 McDonnell JM, Carpenter JD, Jacobs $P$, et al. Conjunctival melanocytic lesions in children. Ophthalmology 1989:96:986-93.

3 Aoyagi M, Morishima N, Yoshino Y, et al. Conjunctival malignant melanoma with xeroderma pigmentosum. Ophthalmologica 1993;206: 162-7

4 Strempel I, Kroll P. Conjunctival malignant melanoma in children. Ophthalmologica 1999;213:129-32.

5 Zhang S, Anderson DF, Bradding P, et al. Human mast cells express stem cell factor. J Pathol 1998;186:59-66.

6 Hartmann ML, Piliponsky A, Temkin V, et al. Human peripheral blood eosinophils express stem cell factor. Blood 2001;97:1086-91.

7 Yanoff M, Fine B. Ocular melanocytic tumors. In: Ocular pathology, a text and atlas. 3rd ed. Philadelphia: JB Lippincott, 1989:631-9.

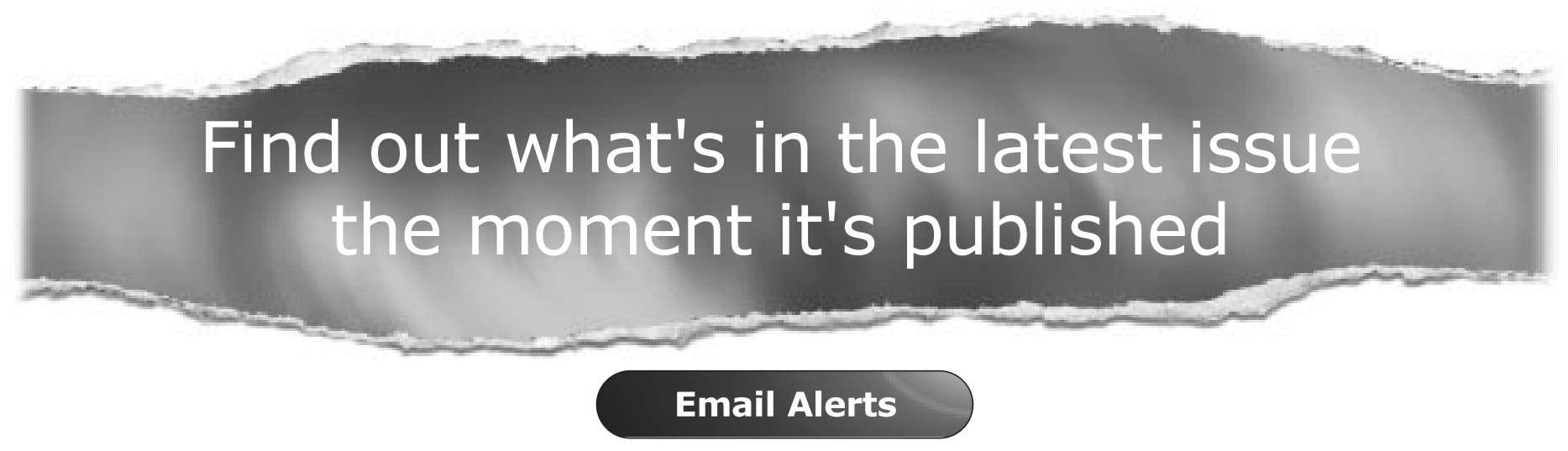

Sign up to receive the table of contents by email every month. You can select from three alerts: Table of Contents (full), TOC Awareness (notice only); British Journal of Ophthalmology related announcements.

\section{wwww.bjophthalmol.com}

